\title{
Destinazione turística. Quando la popolazione diventa un "atrattore"
}

\author{
Tiziano Gasbarro
}

\section{SciELO Books / SciELO Livros / SciELO Libros}

GASBARRO, T. Destinazione turística. Quando la popolazione diventa un "atrattore". In: MARAFON, G. J., FACCIOLI, M., and SÁNCHEZ, M. A., ed. Patrimônio, território e turismo no Brasil, Costa Rica e Itália [online]. Rio de Janeiro: EDUERJ, 2020, pp. 117-146. ISBN: 978-6500-03032-7. https://doi.org/10.7476/9786500030327.0006.

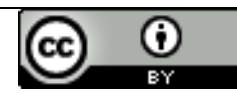

All the contents of this work, except where otherwise noted, is licensed under a Creative Commons Attribution 4.0 International license.

Todo o conteúdo deste trabalho, exceto quando houver ressalva, é publicado sob a licença Creative Commons Atribição 4.0.

Todo el contenido de esta obra, excepto donde se indique lo contrario, está bajo licencia de la licencia Creative Commons Reconocimento 4.0. 


\section{Destinazione turística. Quando la popolazione diventa un "atrattore"}

Tiziano Gasbarro ${ }^{1}$

\section{Introduzione}

Cercare di comprendere ed analizzare il modo in cui, soprattutto ma non esclusivamente, le popolazioni possano e anzi debbano rappresentare un fulcro all'interno della definizione di una destinazione turistica di successo, ponendosi quale discriminante principale nella promozione dell'immagine della destinazione che verrà a crearsi. Questo l'obiettivo che le pagine successive tentano di porsi, in molti casi indicando e riportando fonti alla base di ragionamenti qui citati e che nascono, per l'appunto, da un metodo ed una bibliografia pregressi ampiamente discussa e che non necessita ripetizioni in questa sede.

A questo proposito si può iniziare notando come sempre più frequentemente ricorre il concetto di "creazione" della destinazione turistica. Altrettanto spesso non si riesce a comprendere quali elementi dovrebbero necessariamente entrare a farne parte né, nei casi in cui tale destinazione esista già, in che modo sarebbe opportuno farla progredire, evitando stagnazione sociale e, molto più importante, imprenditoriale. Emerge a questo proposito la necessità, prima di tutto, di comprendere dove una determinata destinazione voglia posizionarsi rispetto al target di riferimento. Nel caso specifico compete un posizionamento focalizzato "nel definire l'offerta e l'immagine di un'impresa ${ }^{2}$ in modo da consentirle di occupare una posizione distinta

1 Tiziano Gasbarro è Dottorando in Beni Culturali, Formazione e Territorio (XXXII ciclo), presso l'Università degli Studi di Roma “Tor Vergata”. Geografo Politico-Economico si interessa di Aree Naturali Protette, pianificazione territoriale e rigenerazione in chiave sostenibile sia urbani che extraurbani.

2 Il riferimento all'impresa non è un refuso. La scelta è quella di trattare la destinazione turistica come un prodotto generato da un insieme di enti, pubblici e privati, che collaborano verso il raggiungimento di un obiettivo comune, volto alla realizzazione di una risultate aziendale 
e apprezzata nella mente del mercato obiettivo" (Kolter, 2004, p 372). Si intersecano i concetti di posizionamento, essenziale nel processo turistico, e di immagine. Tali attributi permettono di stabilire, verso il target, esattamente il ruolo che si vuole trasmettere. L'immagine acquisisce allora un ruolo quale l'essere un fattore di successo delle destinazioni turistiche, cruciale nella promozione, nella distribuzione e nello sviluppo dei prodotti turistici (Sonmez ig Sirakaya, 2002; Pikkemaat, 2004).

\section{Prospettive di destinazione}

Secondo l'Organizzazione Mondiale del Turismo (OMT) una destinazione turistica è definita come

a physical space in wich a visitor spends at least one overnight. It includes tourism products such as support services and attractions, and tourism resources within one day's return travel time. It has physical and administrative boundaries defining its management, images and persceptions defining its market competitiveness. Local tourism destinations incorporate various stakeholders often including a host community, and can nest and network to form larger destinations.

Diventa necessario, prendendo a riferimento tale definizione, tenere in considerazione una serie di caratteristiche e modificazioni alle strutture territoriali, così come sopra introdotte. La pianificazione di spazi fisici e sociali si compone, oltre che di elementi concreti, di azioni “intangibili”. Essenziale si dimostra la capacità di accrescimento dell'area d'intervento, non solamente spaziale ma anche qualitativo, ponendo alla base strategie di destination management. Peculiarità del settore turistico è la capacità di generare valore per la destinazione non soltanto in prospettiva di flussi turistici ma anche, e soprattutto, nelle possibilità di produrre risorse potenziali per la collettività. Ad esempio, la relazione tra beni culturali e turismo viene oggi sempre più interpretata come una reazione produttiva in cui gli stessi - dopo un lungo periodo durante il quale sono stati considerati solo come patrimonio da preservare - assumono il ruolo

di successo. Nelle pagine successive verrà indicato più nello specifico quali passi sono stati individuati opportuni e maggiormente aderenti alle idee e proposte attuabili. 
di "attrattori turistici" (Simeon, 2000) e diventano fattori di sviluppo locale (De Varine, 2005; Greffe, 2003; Martelloni, 2006). Ma non si tratta solamente di potenziare il patrimonio culturale materiale, come abbiamo visto, bensì di crearne altrettanto, se non di più, di immateriale ed ancor più delocalizzato.

\section{Agenti di formazione dell'immagine (Gartner, 1993).}

Nel processo di produzione d'immagine esistono molteplici canali che, nel corso degli anni, hanno subito una ridefinizione non nella struttura quanto nella tipologia. Quello che cambia non sono i contenuti ma i mezzi di diffusione. L'avvento dei social media e degli influencer ne diventa il caso emblematico. Nello specifico, trattando la creazione di una destinazione turistica di successo, si ritiene assolutamente valido un discorso improntato sull'utilizzo di:

- Fonti informative indotte palesi di primo livello: forme tradizionali di pubblicità. Si sa che l'emittente vuole promuovere la destinazione.

- Fonti informative indotte palesi di secondo livello: tour operator, agenzie di viaggio ecc... la promozione concorre alla percezione che si costruisce nell'immaginario del turista.

- Fonti informative indotte non palesi di primo livello: tradizionali fonti ma accompagnate da una figura di spicco che ne esalta le qualità.

- Fonti informative indotte non palesi di secondo livello: articoli, reportage, storie relative ad un determinato posto proveniente da una fonte apparentemente non interessata allo sviluppo turistico della destinazione. Credibilità data dal fatto che le persone sembrano estranee alle dinamiche territoriali.

- Fonti informative autonome: report, documentari, film e articoli sulla località.

- Fonti informative organiche non sollecitate: divulgazione di informazioni da parte di chi è stato in visita nel luogo.

- Fonti informative organiche sollecitate: il processo di ricerca attivato da chi sceglie di partire, indeciso tra più destinazioni. Il passaparola è fortemente considerato.

- Fonti organiche: informazioni raccolte postume alla visita dell'area. 
La public policy ${ }^{3}$ si interessa con sempre maggior vigore alla costruzione di processi di governance del territorio, definendo modalità di coordinamento delle dinamiche economiche e sociali mediante il coinvolgimento di un numero sempre maggiore, e con estrazione sempre più diversificata, di attori. Cambia nella sostanza l'approccio che si ha verso la proposizione di un prodotto turistico (good-dominant $\operatorname{logic}{ }^{4}$ ) ponendo l'attenzione sulla vendita di servizi e, ancor di più, esperienze. Ci si trova in uno stato di service-dominant logic (Vargo e Lusch, 2004; Lusch e Vargo, 2006), all'interno del quale si mettono

3 Ho deciso, nell'ambito di questo contributo, di escludere la percezione di una destinazione turistica attraverso il ciclo di vita descritto da Butler (1980), da lui suddiviso in fasi fondamentali: 1) Esplorazione; 2) Coinvolgimento; 3) Sviluppo; 4) Consolidamento; 5) Stagnazione; 6) Ringiovanimento o Declino. Alla base delle considerazioni evidenziate nelle pagine successive si trovano piuttosto i contributi, rielaborati grazie al testo citato in bibliografia "Il turismo nelle/delle destinazioni" - e dal quale prendo molti degli spunti che io stesso inserisco nel discorso - di alcuni tra gli autori che più assiduamente si sono occupati, e si occupano, di trattare la public policy italiana. Faccio riferimento a pubblicazioni quali Conti S., Salone C., Programmazione integrata e politiche territoriali. Profili concettuali, esplorazioni progettuali, IRES Piemonte, Torino, 2011; Conti S., Sforzi F., Il sistema produttivo italiano, in Coppola P. (a cura di), Geografia politica delle regioni italiane, Einaudi, Torino, pp. 278-336, 1997; Conti S., Territori industriali: imprese e sistemi locali, in "Geotema” 2, Pàtron, Bologna, 1995; Conti S., Giaccaria P., Rossi U., Salone C., Geografia economica epolitica, Pearson Italia, Milano, 2014. Come ho tentato e tenterò di evidenziare, sarà proprio il ruolo attribuito ed attribuibile alle figure pubbliche del tessuto territoriale di volta in volta selezionato, a porsi in risalto ed in linea con le modificazioni delle prospettive dell'offerta e della domanda turistica in relazione alle destinazioni createsi. Questo non significa l'abbandono di standard o dinamiche precedentemente valide ma una loro rielaborazione ed un loro ripensamento ed inquadramento nelle realtà moderne. A questo proposito ho scelto di focalizzare l'attenzione solo su di una parte individuala quale funzionale alla mia breve, e forse poco approfondita, analisi delle dinamiche in un territorio in mutazione; questo non a discapito dell'efficacia che tali interventi dimostrano all'interno dei contesti nei quali sono stati, o saranno, applicati.

4 Dal Town Center Management britannico alla Galerie Merchande à ciel ouvert francese, passando per i Centro comerciales abiertos o urbanos spagnoli, in tutta Europa si registra una diffusa tendenza a rilanciare l'attività commerciale delle aree centrali attraverso azioni di city branding, networking, destination management e benchmarking tipiche delle strategie territoriali di matrice turistica (Cirelli, Nicosia, 2013). 
a disposizione dei clienti, dei turisti, "risorse", materiali e immateriali, affinché concorrano responsabilmente alla creazione di valore. A mettere in crisi, volendo usare un termine forse troppo duro ma certamente efficace a riprova della modificazione della logica di mercato, il modello poc'anzi citato, è sicuramente la possibilità di accedere ad un bacino di conoscenza ${ }^{5}$ estremamente più ampio rispetto alla situazione pregressa; non più soltanto una delle tante e diversificate risorse ma "la caratteristica essenziale, l'elemento fondante dell'esistenza dell'impresa e del processo di creazione del valore” (Vicari, 2008, p. 54). È interessante constatare come in "un capitalismo costruito sul lavoro della mente, il valore è generato proprio trasformando e utilizzando pensieri, emozione, identità" (Rossi, 2014, p. 17). Emerge nuovamente l'importanza della collaborazione, dell'ampliamento delle interazioni tra gli stakeholders diretti ed indiretti di una destinazione. Esiste però un problema che si pone nella presenza sempre più massiccia della rete $^{6}$. Le informazioni diventano certamente più accessibili, ma questo comporta il pericolo di uno scavalcamento per alcune delle figure, fino ad ora ritenute essenziali, nella filiera turistica: tour operator ed agenzie dettaglianti. Fortunatamente sempre più enti e strutture, incluse le figure già citate, vedono nella rete un alleato piuttosto che un pericoloso rivale; preferiscono rimodulare parte della loro offerta seguendo le tendenze che dallo stesso mercato vengono imposte. Cambiano, di conseguenza, le logiche legate al mercato esaminato. Nell'ottica di una logica di servizio diventa preponderante la ricerca dell'esperienza autentica ${ }^{7}$, incontaminata - ovviamente dal punto di vista della percezione dell'utente e dalla capacità degli attrattori di una destinazione di venderla come tale - volgendo l'attenzione alla capacità che gli stakeholders

5 L'economia della conoscenza non riguarda i processi cognitivi della singola impresa, ma quelli della filiera cognitiva, ossia dell'insieme di operatori che si scambiano la conoscenza attraverso le diverse fasi della catena, passando dalla produzione, alla propagazione e all'uso (Rullani, 2004, p. 27).

6 L'estrazione di chi utilizza internet non è più strettamente legata all'età, così come il mercato si sta spostando sulla rete e non la ritiene più un rivale quanto un punto di forza del quale prendere coscienza.

7 Gli attributi che maggiormente definiscono una destinazione come autentica sono: il fatto che il luogo rimanga inalterato nel tempo, che i suoi paesaggi siano unici e non replicabili, che la comunità locale sia genuina e ospitale e, infine, che la cultura e le tradizioni siano uniche e fortemente presenti (Atzeni et al, 2018). 
avranno nel far sì che la stessa mantenga le aspettative con le quali il turista si approccia al prodotto finale, riuscendo nel migliore dei casi ad accrescerle. Il viaggiatore ricerca, infatti, la possibilità di fare esperienze che rispecchino i luoghi, interagendo con le persone - e nuovamente emerge, qui, come la componente socio-culturale di un luogo si riveli essenziale alla creazione di una destinazione turistica credibile e di successo - arrivando a percepire un processo di crescita interiore, fino al raggiungimento di valori terminali come la felicità (Atzeni et al, 2018), l'autorealizzazione e la crescita del proprio io (Berman, 1970).

\section{Governo e creatività dei territori}

Facendo riferimento ad un territorio che punta a divenire una destinazione turistica, serve individuare un organismo che sia espressione "dell'integrazione e del coordinamento delle decisioni che afferiscono agli attori territoriali” (Golinelli M. C. et al, 2006, p. 26). Per far sì che questo accada, è necessario che si passi da una forma di gestione amministrativa dei fatti locali (governement) ad una che tenga fortemente in considerazione gruppi sociali ed istituzioni, decentralizzando dinamiche economiche e sociali (governance) (Sabel, 1998). Secondo Rullani (2002) il passaggio dal government alla governance porta con sé una doppia trasformazione:

- l'attenzione si sposta dalla struttura al processo;

- si passa da un approccio esclusivamente di governo (pubblica amministrazione) ad un altro basato sulla valorizzazione delle capacità civiche e delle politiche di volontariato.

Non sembra essere dello stesso avviso, però, Bonomi ${ }^{8}$, il quale percepisce una "centralizzazione e verticalizzazione dei poteri di cui il drastico accompagnamento delle province è solo il più evidente degli aspetti” che interessano il microcosmo produttivo italiano, ritenuto principale destinatario del passaggio da government a governance nelle modalità appena descritte 9

\footnotetext{
8 Bonomi A., Se il territorio è in ebollizione, in "Il Sole 24 Ore”, 19 agosto 2012

9 Il modello del territorio del quale parla Bonomi è quello "autopoietico". il riferimento è all'idea di sistema autopoietico introdotta da H. Maturana e F. Varela, e cioè a quella "unità composita la cui organizzazione può essere descritta come un reticolo chiuso di produzioni
} 
Tali considerazioni, anche se più recenti, sembrano non tener conto di come, ormai, una destinazione turistica venga indentificata tanto dagli operatori quanto dagli utenti. Concretamente, una destinazione è rappresentabile come una "confezione" (Leiper, 1990) di prodotti e servizi parzialmente creati da operatori pubblici e privati che pongono il focus non sulla singola attrazione, ma sulla combinazione di fattori attraverso i quali avviene l'esperienza di viaggio del turista. Potremmo anche dire che si qualifica la destinazione solo se esiste una "offerta parzialmente determinata che si fonda su un aggregato di risorse, attività e attori, il cui funzionamento deve interpretarsi come un sistema" (Tamma, 2002, p. 12). Lo stesso Tamma (2002, p. 17) individua le componenti rappresentative di una destinazione in:

- Uno spazio geografico definito;

- Un riferimento congiunto ad un'offerta (magari articolata per rispondere alle differenti esigenze del mercato) e a un mercato (rappresentato in termini di segmenti differenti l'uno dall'altro);

- L'insieme delle risorse - che in base a quanto visto in precedenza possiamo distinguere in operand (naturali, artificiali, ecc) e operant (attori e loro know-how) secondo la prospettiva della SDL - utilizzabili per l'erogazione dell'offerta.

Concordemente a questi elementi di modifica nelle dinamiche della gestione del territorio di una destinazione, indispensabile si pone l'individuazione di pratiche che sappiano aggregare e promuovere efficacemente le risorse rivolte al turista. L'attenzione si pone sul Destination Management/Marketing Organization (DMO) che, prendendo a riferimento la definizione, rappresenta:

the leading organizational entity which may encompass the various authorities, stakeholders and professionals and facilitates tourism sector partnershis towar$\mathrm{d}$ a collective dstination vision. The governance structures of DMOs vary form a single public authority to a public/private partnership model with the key role

di componenti che, attraverso le loro interazioni, costituiscono essi stessi il reticolo di produzioni che li produce e ne determinano l'estensione generandone i confini del loro dominio d'esistenza" (Maturana, 1993, p. 59). Tale modello, recentemente, può ritenersi ampiamente superato o, laddove ancora persistente, in crisi. 
of initiating, coordinating and managing certain activities such as implementation of tourism policies, strategic planning, product development, promotion and marketing and convetion bureau ativities. The functions of the DMOs may vary from national to regional and local levels depending on the current and potential needs as well as on the decentralization level of public administration. Not every tourism destination has a $\mathrm{DMO}^{10}$.

Si mette subito in evidenza come le funzioni di management e quelle di marketing facciano capo alla stessa organizzazione. Questo non preclude la coesistenza di molteplici modelli organizzativi delle $\mathrm{DMO}^{11}$, tanto che si pone l'accento su come queste possano comprendere autorità pubbliche, imprese, professionisti e altri portatori di interesse operanti in ambito turistico che vogliano cooperare nella prospettiva di una visione collettiva della destinazione. La necessità di coesione d'intenti e collaborazione è anche qui sottolineata, rivolgendosi a tutte le parti interessate, ponendo sullo stesso livello d'importanza tanto le ormai radicate aziende di settore quanto i nuovi attori del turismo, primi tra tutti i privati, i volontari; in sostanza, le popolazioni. Una volta in più appare evidente che le decisioni legate alla creazione di una destinazione turistica di successo "non possono essere compiutamente assunte se non alla luce delle attese della moltitudine di stakeholder che a differente titolo partecipa ai processi di creazione di valore (Napolitano, 2010; Napolitano e De Nisco, 2007).

Nel paragrafo precedente si è accennato al ruolo che i beni culturali hanno sempre avuto e che, più di recente, stanno assumendo nel contesto di creazione di una destinazione turistica. Congiuntamente si è fatta menzione della componente esperienziale, intangibile, creativa. Questa componente subentra con l'ingresso, tra i decisori, di figure intensamente legate al territorio che si prende a riferimento. Ma chi sono i protagonisti di questo binomio cultura-

${ }^{10} \mathrm{http}$ //destination.unwto.org/content/conceptual-framework-0

11 Ad oggi, le DMO e gli enti delle destinazioni sono di fatto degli attori esterni al sistema turistico della destinazione stessa. Lavorano parallelamente agli enti già presenti sul territorio ed in alcuni casi incidono positivamente, ricordandoci però come in molti altri casi, invece, non riescano né a dialogare né a rappresentare il sistema turistico. "Sono spesso castelli arroccati in sé stessi, scollegati dalla realtà del settore e soprattutto dai turisti” (Ejarque, 2016). Ovviamente la valutazione caso per caso è la discriminante essenziale per la comprensione di un territorio, sia in caso di esempi di successo che di insuccesso. 
-creatività? Change makers, urban regenerators, smart citizens, sono questi agli "innovatori" delle città, protagonisti di pratiche virtuose, su larga e piccola scala, a volte fuoriuscendo dal contesto "generante" interconnettendo più realtà, amplificandole, ideandone di nuove e più appetibili per un mercato che, sempre più di frequente, si crea nel momento stesso in cui la loro arte prende vita.

Il patrimonio culturale e le sopracitate performing arts, rappresentano settori sani dell'economia reale che ingenerano ritorni sul territorio in termini di ricchezza prodotta, occupazione, nascita di nuove imprese, forza e durata delle relazioni tra soggetti. In ambito turistico rappresentano il valore aggiunto, portando creatività ed innovazione, favorendo e migliorando l'incontro tra la nuova domanda di esperienze turistiche - come è stato già più volte sottolineato fin qui - e un'offerta corrispondente di servizi. Per comprendere e meglio valorizzare queste nuove e mutevoli risorse territoriali è necessario restringere il campo attualizzando e trasformando le più tradizionali formule di fruizione turistica culturale. 


\section{Generazione di esperienze (Pine e Gilmor, 1999)}

Il consumo turistico nell'era della creatività abbraccia la necessità di diventare scenario per la generazione e l'effettuazione di esperienze. La creatività implica un nuovo modo di rapportarsi all'esperienza di viaggio. L'esperienza creativa permette infatti al turista di entrare in relazione con altri soggetti e di avere un contatto reale con la comunità e la cultura locale.

La creatività, allora, contribuisce allo sviluppo delle destinazioni:

Adottando pratiche non convenzionali, poggiati sulla valorizzazione di aspetti dell'heritage locale, forti di un'attrattività non ancora sviluppata;

Attuando gli effetti di commodification e globalizzazione culturale sulle risorse del territorio;

Stimolando nuove opportunità di sviluppo occupazionale specie per le nuove professioni turistiche;

Accogliendo nuovi pubblici di visitatori;

Avendo un effetto moltiplicatore sull'economia del territorio, attivando collaborazioni professionali ma anche un vero e proprio indotto economico-commerciale;

Fornendo nuove forme di promozioni culturale, creando continuità di followers, legati al territorio e alla sua cultura;

Favorendo il passaggio dall'isolamento all'integrazione delle piccole e medie imprese, agendo positivamente nei confronti dei piccoli operatori che, nei circuiti turistici tradizionali, hanno poche possibilità di inserimento.

Scheda 2

La creatività sembra allora essere fonte di contribuzione essenziale, con specifici riferimenti a quei territori caratterizzati da una prevalenza di piccole e medie imprese. L'azione si focalizza sulla circolazione e diffusione di conoscenze, professionalità, stimoli e innovazioni, come già evidenziato precedentemente. L'introduzione nel contesto reticolare degli stakeholders di un territorio può e deve allargare le possibilità di un singolo, in particolar modo tenendo in considerazione la presenza di privati non riuniti in associazioni o categorie di settore, attraverso progetti che hanno effetti nel lungo periodo e che possono 
essere replicati, plasmandosi sulla base delle esigenze e delle preferenze emerse nel mercato e nei consumatori, dettagliandoli per le specificità dei territori che ne prevedono l'eventuale implementazione.

Le attività di cui alla tabella seguente possono porsi alla base di una implementazione del sistema di misurazione della collaborazione tra gli attori di una destinazione turistica, modulandosi, come visto, alle esigenze specifiche del caso in esame. ${ }^{12}$

\section{Dimensioni e item del modello DART}

\begin{tabular}{|c|c|}
\hline \multirow{6}{*}{ 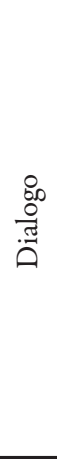 } & $\begin{array}{l}1 \text { Utilizzare canali di comunicazione diversificati per avere sessioni di dialogo con i } \\
\text { consumatori }\end{array}$ \\
\hline & 2 Condurre sessioni di dialogo con il consumatore frequentemente \\
\hline & $\begin{array}{l}3 \text { Coinvolgere le unità organizzative interne durante le sessioni di dialogo con i } \\
\text { consumatori }\end{array}$ \\
\hline & $\begin{array}{l}4 \text { Coinvolgere stakeholder esterni all'organizzazione durante le sessioni di dialogo con i } \\
\text { consumatori }\end{array}$ \\
\hline & $\begin{array}{l}5 \text { Riconoscere il contesto di esperienza sociale, emozionale e culturale del consumatore } \\
\text { riguardo al prodotto/servizio }\end{array}$ \\
\hline & 6 Sottolineare lo sforzo dei dipendenti verso i singoli consumatori \\
\hline \multirow{5}{*}{ 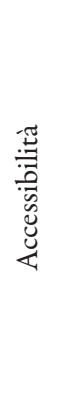 } & $\begin{array}{l}1 \text { Offrire ai consumatori la possibilità di condividere il processo di progettazione del } \\
\text { prodotto di servizio }\end{array}$ \\
\hline & $\begin{array}{l}2 \text { Offrire ai consumatori la possibilità di condividere il processo di sviluppo del prodotto } \\
\text { di servizio }\end{array}$ \\
\hline & $\begin{array}{l}3 \text { Offrire l'opportunità ai consumatori di condividere il processo di fissaggio dei prezzi del } \\
\text { prodotto di servizio }\end{array}$ \\
\hline & $\begin{array}{l}4 \text { sottolineare maggiormente la necessità di fornire esperienze ai consumatori rispetto alla } \\
\text { proprietà del prodotto di servizio }\end{array}$ \\
\hline & 5 Fornire ai consumatori tutte le informazioni necessarie sul prodotto/servizio \\
\hline
\end{tabular}

12 Il modello DART rappresentato in tabella fa riferimento ad un sistema economico più ampio, spesso indirizzato specificatamente ad una analisi di vendita di prodotto. Ho però ritenuto tale modello perfettamente coerente con la costruzione e produzione di una destinazione turistica, assimilando la stessa, per l'appunto, ad un prodotto da vendere. In questo modo vengono tenuti in considerazione aspetti ed azioni intraprese e da intraprendere grazie al coinvolgimento della pienezza degli attori presenti sul territorio. La coesione e collaborazione in un "sistema rete" tornano ad essere alla base di tutte le proposte possibili ed attuabili, al netto della considerazione dovuta agli elementi presenti nel modello. 


\begin{tabular}{|c|c|}
\hline \multirow{5}{*}{ 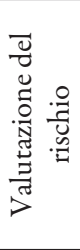 } & 1 Informare chiaramente i consumatori circa il servizio offerto \\
\hline & 2 informare i consumatori sui limiti riguardanti le conoscenze e le capacità dell'impresa \\
\hline & 3 riconoscere le mutevoli dinamiche dei bisogni dei consumatori \\
\hline & 4 Accettare le lamentele dei consumatori riguardanti la proposta di valore \\
\hline & 5 Accollarsi le responsabilità dei rischi derivanti dall'attività di impresa \\
\hline \multirow{5}{*}{ 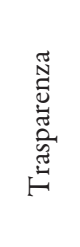 } & 1 Rendere chiare ai consumatori le informazioni relative al prodotto/servizio \\
\hline & 2 Divulgare tra i consumatori le informazioni relative ai prezzi \\
\hline & 3 Trarre benefici dalla simmetria informativa tra i consumatori e l'impresa \\
\hline & 4 Costruire la fiducia tra i consumatori attraverso informazioni trasparenti \\
\hline & 5 Fornire informazioni aggiornate ai consumatori \\
\hline
\end{tabular}

Fonte: Traduzione da Taghizadeh, Jayaraman, Ismail e Rahman (2016)

\section{Elementi e risorse per uno sviluppo di successo}

Abbiamo visto fin qui come per destination management si intenda la gestione strategica di un territorio che voglia essere destinazione turistica, e che pertanto faccia "riferimento all'insieme di decisioni, di strumenti e di approcci gestionali che configurano la vita di una destinazione nella sua complessità, a cura della moltitudine di soggetti e/o organizzazioni (manager, imprenditori, policy maker, ecc) che partecipano, a differente titolo, alla sua definizione" (Napolitano, 2010). Analogamente possiamo definire il destination management quale un sistema di gestione strategica del territorio basato su appropriati sistemi di pianificazione e controllo delle attività da sviluppare, così che i flussi turistici presenti in un'area possano essere oggetto di ulteriore incentivo (Della Corte e Sciarelli, 2000).

Un problema si pone però nel momento in cui bisogna individuare in che modo una organizzazione, una gestione mirata, possa favorire sviluppo e collaborazione. Per far questo si possono individuare alcuni attori preesistenti, agevolando in questo modo la successiva introduzione di nuovi e più moderni. A tale proposito, Thomas e Wood (2014), considerando uno studio nel settore alberghiero. Da questo è possibile partire per analizzare e definire le capacità di assorbimento di una determinata destinazione, sotto uno dei 
punti ritenuti essenziali, quale si dimostra essere l'ospitalità del potenziale turista. $^{13}$

\section{Tabella capacità di assorbimento e relativi item nel campo del settore al- berghiero}

\begin{tabular}{|l|c|c|}
\hline \multicolumn{2}{|c|}{ Capacità } & Determinanti \\
\hline \multirow{4}{*}{} & \multirow{4}{*}{ Acquisizione } & Incontri con terze parti \\
\cline { 3 - 3 } & & Rispetto reciproco \\
\cline { 3 - 3 } & & Reciproca fiducia \\
\cline { 3 - 3 } & & Interazione personale \\
\cline { 3 - 4 } & \multirow{3}{*}{ Assimilazione } & Capacità di assimilare innovazioni e nuovi processi tecnologici \\
\cline { 3 - 4 } & & Abilità nell'utilizzare le conoscenze e le esperienze dei \\
& & dipendenti \\
\cline { 3 - 4 } & & $\begin{array}{c}\text { Abilità nell'assimilare conoscenza dall'esperienza di successo } \\
\text { di altre organizzazioni operanti nello stesso ambito industriale }\end{array}$ \\
\hline
\end{tabular}

13 Un doveroso appunto va fatto sul modello dell'offerta turistica dal lato dell'ospitalità. Per quanto lo studio risulti meticoloso, non bisogna dimenticare il notevole impulso dato al settore da parte di soluzioni alternative alla classica offerta alberghiera. Essenziale si dimostra la presenta di strutture complementari, aggregate sempre più di frequente e con sempre maggior efficacia attraverso portali dedicati. Basta osservare l'offerta portata da AirBnB, Booking (anche quest'ultimo ha iniziato, in anni recenti, a diversificare l'offerta, comprendendo case vacanza, seconde case o situazioni d'alloggio quali hostelli, affittacamere e campeggi organizzati in piazzole munite di bungalow), per capire come la rinascita di una destinazione possa, e forse debba, passare attraverso strutture e servizi preesistenti ai quali venga modificata, con cognizione, la destinazione d'uso. 


\begin{tabular}{|c|c|c|}
\hline \multirow{8}{*}{ 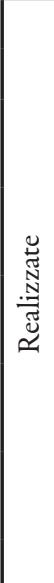 } & \multirow[t]{5}{*}{ Trasformazione } & Capacità di coordinare e integrare le conoscenze \\
\hline & & Incoraggiare i dipendenti alla condivisione delle informazioni \\
\hline & & $\begin{array}{l}\text { Abilità nell'uso delle ICT per migliorare i flussi informativi e } \\
\text { le comunicazioni }\end{array}$ \\
\hline & & $\begin{array}{l}\text { Consapevolezza del bisogno di usare nuove conoscenze per } \\
\text { poter innovare }\end{array}$ \\
\hline & & $\begin{array}{l}\text { Rapida applicazione delle nuove conoscenze riconosciute } \\
\text { come importanti }\end{array}$ \\
\hline & \multirow[t]{3}{*}{ Sfruttamento } & $\begin{array}{l}\text { Riconsiderare la tecnologia utilizzata e adattarla alle nuove } \\
\text { conoscenze }\end{array}$ \\
\hline & & $\begin{array}{l}\text { Capacità di usare le nuove conoscenze per rispondere } \\
\text { tempestivamente alle sollecitazioni del mercato }\end{array}$ \\
\hline & & Abilità nell'innovare per guadagnare in competitività \\
\hline
\end{tabular}

Fonte: Traduzione da Thomas \& Wood, 2014, p. 45

Quanto in tabella può riferirsi a situazioni analoghe, tanto per forme di ospitalità quali strutture extralberghiere classiche (campeggi, resort ecc...) quanto per le nuove, rappresentabili nel mercato quali esclusivamente pertinenti al mercato on-line (AirBnB, Booking, Expedia ecc...).

Con le medesime finalità dello studio svolto da Thomas e Wood, si è portata avanti un'indagine ${ }^{14}$ atta ad individuare i principali interessi dei turisti che usufruiscono di tour operator - altro ente del territorio che, idealmente, promuove e vende nel migliore dei modi, con il massimo delle conoscenze, un territorio - è stata sottoposta ad enti associati, francesi e inglesi. ${ }^{15}$

Attraverso il confronto tra indagine e letteratura, sono emersi 17 attributi che presentano un valore rilevante:

- Posti di interesse storico e culturale

- Attrazioni naturali

14 Fonte dell'indagine è il Programma esecutivo annuale disponibile su www.enit.it per l'anno 2005. Ho ritenuto utile basarmi sull'indagine del 2005 poiché i dati analizzati risultano più aderenti al contesto di riferimento nel contributo da me portato. Si raccomanda comunque per completezza la consultazione dei report per gli anni successivi, così da poter osservare l'evolversi della situazione, esaminandone i risultati delle azioni proposte ed attuate nel corso degli anni.

15 Cellini R., Il turismo nei programmi di crescita e sviluppo: analisi teoriche ed esperienze, in La Rosa R. (a cura di), Lo sviluppo del turismo in Sicilia, Franco Angeli, Milano, 2004 
- Presenza di negozi e centri commerciali

- Presenza di strutture sanitarie

- Strutture ricettive di alta qualità

- Strutture ricettive con un numero elevato di posti letto

- Facilità di accesso alla destinazione

- Possibilità di praticare attività sportive

- Vivace vita notturna e divertimenti

- Buona cucina

- Professionalità dei tour operator locali

- Sicurezza pubblica

- Buon rapporto qualità/prezzo dei servizi turistici

- Pulizia e igiene dell'ambiente

- Ospitalità e gentilezza dei residenti

- Efficienza dei servizi turistici

- Qualità e quantità della promozione e dell'informazione turística

Sorprende il fatto che gli operatori di entrambi i paesi citino tra i fattori di appeal la presenza di vulcani e isole vulcaniche - l'elemento è emerso alla voce "Attrazioni naturali"; la Sicilia, come anche Napoli, Ponza e le altre isole lungo la medesima fascia costiera, sembrano dunque nella condizione di poter essere percepite come "altro" rispetto alla forma di turismo tradizionale che comprende generalmente mare e cultura - e questo possa comportare non soltanto una diversificazione e destagionalizzazione della destinazione turistica, ma l'approntamento di veri e propri servizi e corsi di formazione per lo sviluppo e la diffusione di questo segmento turistico, entrando per l'appunto nelle campagne di promozione di enti quali i tour operator in una visione più ampia dell'offerta turistica integrata nel management dei territori.

Individuare gli attori che, sinergicamente, possano dare vita ad una destinazione turistica di successo necessita, nella fase successiva, di comprendere come, su quali basi, debbano procedere. A questo proposito è possibile circoscrivere una serie di steps applicabili, su scale anche differenti e su realtà non troppo distanti concettualmente tra loro, attraverso i quali non solo la partecipazione degli stakeholders viene incentivata ed amplificata, ma permettono anche e soprattutto l'ampliamento delle categorie di figure interessate a subentrare nella gestione del territorio. 
Progressivamente si porrà un focus su:

- La creazione dell'immaginario. L'attività di costruzione dell'immaginario trae particolare beneficio dagli strumenti che favoriscono la narrazione del territorio (guide, mappe, foto, webcam, video, racconti, leggende, fiction tv, ecc...). Accenno è stato fatto, in parte, nella Scheda1;

- Il territorio e le sue rappresentazioni. Si tratta cioè di costruire il prodotto globale (Casarin, 2007) valorizzando il patrimonio già presente nel territorio (ambiente naturale, architetture delle varie epoche storiche, eventi e manifestazioni culturali, ecc...), garantendone la godibilità, la sicurezza e la bellezza dei luoghi, in termini di accessibilità, infrastrutture, informazioni, accoglienza. L'attenzione dovrà mantenersi sull'esperienza del viaggiatore (per esempio la conoscenza delle lingue straniere non soltanto da parte degli albergatori);

- Il viaggiare e il suo sistema di relazioni. Come già visto, la necessità di comprendere cosa il turista scelga di esperire è fondamentale. Osservarli, ascoltarli, capirne i bisogni, le aspettative, i sentimenti e le emozioni con l'intento di realizzare un contesto accogliente in tutti gli ambiti di interazione con gli stessi, condividendo le proprie esperienze positive e negative, nonché le preoccupazioni e le modalità con cui eventualmente le hanno superate e risolte;

- Il concept esperienziale e le modalità di svolgimento. Si tratta cioè di definire quale modus operandi che permette agli attori chiamati ad allestire la proposta di valore di interrogarsi senza soluzione di continuità circa l'adeguatezza della proposta rispetto alle mutevoli istanze provenienti dai viaggiatori;

- Gli attori dell'esperienza turistica. Gli attori fondamentali dell'esperienza turistica sono altri viaggiatori, operatori dei servizi (dell'accoglienza, del trasporto, dell'intrattenimento, ecc...), istituzioni, comunità ospitanti, e, come abbiamo visto con l'avvento delle nuove forme mediali, gli influencer;

- Il sistema dell'informazione riguardante l'esperienza turistica. Il sistema dell'informazione, centrato necessariamente sulle diverse tipologie di viaggiatori, deve poter prevedere anche livelli diversi di 
accesso alle informazioni, nell'ottica di un destination management efficiente;

- Il sistema dell'accoglienza estesa e la nuova filiera turistica. La formazione di personale turistico all'avanguardia, sotto ogni punto di vista e funzione, realizzando una "cultura dell'accoglienza" in tutti gli attori territoriali. Questo comporta un investimento importante in termini di dialogo con tutte le componenti coinvolte, volto a creare maggiore conoscenza, da condividere e ridistribuire tra tutti, fornendo la chiave di lettura univoca per gli obiettivi strategici della destinazione;

- I fattori abilitanti la memoria dell'esperienza. I fattori abilitanti si sono ampliati e assumono forme diverse: tra i souvenir cresce per esempio il ruolo dell'enogastronomia, tra i primi elementi in grado di risvegliare l'esperienza vissuta. Inoltre, alcuni di questi prodotti vengono poi ricercati nei negozi della distribuzione accentuando le possibilità di una reiterazione dell'esperienza da parte del turista.

\section{Esperienze internazionali.}

St. Louis

Un esempio americano di distretto culturale è quello di St. Louis, in Missuri, per molto tempo una città industriale inquinata e poco sicura, tanto che già dagli anni ' 50 molti abitanti avevano abbandonato il centro urbano per trasferirsi in periferia. Nel 1996, partendo dall'iniziativa di un migliaio di volontari, si avviò un processo di coinvolgimento partecipativo dei cittadini alla definizione degli obiettivi di sviluppo futuro della città. A questo primo segnale di progettualità, che venne dal settore no-profit e dalla cittadinanza, si sovrappose l'azione di coordinamento strategico dell'amministrazione pubblica locale. Nacque così il piano St. Louis 2004, che diede vita a più di settanta gruppi di lavoro formati da residenti che si occuparono della definizione ei contenuti del programma che si tradusse poi, immediatamente, in un piano d'azione con scadenze, stanziamenti di risorse ed assunzione di responsabilità che coinvolsero istituzioni pubbliche, imprese private ed associazioni di cittadini. Furono predisposte varie tipologie 
di incentivi fiscali, finanziati ambiziosi programmi pubblici di investimento e create agevolazioni creditizie per l'impresa privata operante nei settori. Il risultato è che oggi St. Louis è tra le aree metropolitane più sviluppate degli Stati Uniti. In particolare, l'area metropolitana di St. Louis è tra le prime al mondo nelle biotecnologie e nelle scienze della vita, e l'agenzia di sviluppo dell'area, la regional chamber \& growth association, progetta sempre nuove opportunità per la localizzazione di nuove imprese e per lo sviluppo della qualità della vita dei lavoratori. In questo processo la cultura permea tutti gli aspetti dello sviluppo urbano di St. Louis: dalla progettazione del sistema dei trasporti pubblici, con il rivoluzionario programma arts in transit nel quale sono coinvolti centinaia di artisti che si arricchisce ogni anno di nuove realizzazioni, all'orientamento professionale dei giovani che vengono coinvolti in programmi formativi tenuti da artisti locali, ma anche di fama nazionale e internazionale, il cui unico scopo è sensibilizzare gli studenti delle scuole medie e superiori nei confronti delle nuove professioni della creatività. La città è inoltre in grado di offrire molte alternative culturali ai suoi abitanti la St. Louis Symphony Orchestra, la seconda orchestra sinfonica del Paese, il Contemporary Art Museum, uno tra i musei più noti al mondo per l'arte contemporanea, il Forest park, uno dei parchi più belli del paese (più grande del Centra park di New York) all'interno del quale si trovano, tra gli altri, il St. Louis Art Museum, il St. Louis Science Center, il St. Louis History Museum e la St. Louis Municipal Opera, ed infine il Laumeier Sculture park, un parco museo con sculture appartenenti ad artisti di fama internazionale che ospita molti degli eventi culturali che animano l'estate e che attirano ogni anno numerosi spettatori ed artisti da tutto il paese. Tali istituzioni puntano sulla partecipazione della comunità locale. Questo è un elemento centrale della loro mission. La St. Louis Symphony ad esempio organizza ogni anno centinaia di concerti gratuiti e numerosi workshop. Tutti i musicisti si occupano delle attività musicali che coinvolgono la città, come suonare nelle chiese, nelle scuole, nei parchi. Il Missuri Botanical Garden è aperto gratuitamente per la cittadinanza, la St. Louis Municipal Opera presenta musical provenienti da Broadway a prezzi che partono d 25 cents. La St. Louis Art Fair, la cui programmazione coinvolge venti strutture culturali collegate, oltra a presentare ogni anno numerose mostre in ambiti diversi, organizza concerti jazz e spetta- 
coli per bambini all'interno di aree a loro dedicate. La presenza pervasiva della cultura nelle varie dimensioni della vita della città dà una grande visibilità alle istituzioni culturali che coinvolgono l'intera cittadinanza nelle attività creando le condizioni ideali per un'ulteriore crescita delle istituzioni stesse: non è un caso, quindi, se a St. Louis, come in tutti gli States, uno dei principali canali di sostegno finanziario alla cultura è quello dei contributi dei privati cittadini.

\section{Ruhr}

Tra le esperienze europee, molto significativa ed originale è quella dell'Emscher landscape park, nella regione della Ruhr in Germania, che consiste nella riconversione di un'ara di oltre $300 \mathrm{kmq}$, distribuita lungo il territorio fluviale dell'Emscher, da area industriale in abbandono a centro turistico e di promozione culturale. Il distretto minerario e siderurgico rappresentava il cuore industriale dell'Europa del secolo scorso. Tra il 1960 e il 1980 una profonda crisi economica dovuta all'innovazione nei modi della produzione industriale ed alla progressiva dismissione degli impianti minerari stava provocando il declino della popolazione. Nel 1989 il Land, il governo regionale della North Rhine-Westphalia, decise di dare vita ad una radicale trasformazione dell'area dell'Emscher con l'obiettivo principale di realizzare un parco naturalistico e paesaggistico in un ambiente caratterizzato da enormi fabbriche e miniere dismesse, fiumi trasformati in canali fognari a cielo aperto, elevati livelli di inquinamento atmosferico e sviluppo urbanistico disordinato. Il Land approvò, quindi, il programma decennale dell'IBA Emscher park e ne stabili gli obiettivi generali che riguardavano sviluppo urbano e misure sociali, culturali ed ecologiche come basi per la trasformazione economica della vecchia regione industriale della Ruhr. La regia dell'intervento fu affidata alla IBA Emscher park, una società consociata a responsabilità limitata di proprietà della regione, preposta alla ricostruzione del paesaggio dell'Emscher. L'IBA non ebbe potere giuridico-legale né costituì un'autorità per la pianificazione, ma si configurò come un'agenzia di consulenza con lo scopo di realizzare una progettazione concertata e partecipata con i numerosi gruppi sociali, ambientalisti ed imprenditoriali presenti nell'area. In questo quadro complesso la società svolse il ruolo di moderatore e spesso di incubatore dei progetti ma la responsabilità dei singoli interventi 
restò ai titolari dei contratti, le autorità locali ma anche le imprese ed i soggetti privati. Per realizzare gli interventi per l'IBA Emscher park non vennero utilizzate fonti di finanziamento straordinarie. Le azioni di pianificazione furono finanziate da programmi di aiuto alla regione già esistenti, combinati a fondi strutturali statali e comunitari. Durante la fase IBA vennero investiti nella regione cinque miliardi di marchi, di cui circa due terzi provenienti da fonti di finanziamento pubblico ed un terzo da investimenti privati. Il programma di riqualificazione del paesaggio della Ruhr promosso dall'IBA dal 1989 al 1999 si fondava su sette obiettivi specifici: la ricostruzione del paesaggio dell'Emscher; il riassetto idrogeologico del sistema fluviale dell'Emscher; il recupero del Rhei-herne-kanal come spazio ricreativo; il recupero dell'archeologia industriale come testimonianza storica; nuovi posti di lavoro nel parco; nuovi spazi abitativi e forme innovative dell'abitare; a promozione di attività sociali, culturali e sportive. Le procedure di selezione degli interventi nell'Emscher park si basarono su nu sistema concorsuale che valutò, essenzialmente, la qualità estetica, ecologica ed ambientale e la fattibilità concreta dei progetti presentati. Nei dieci anni di fase IBA furono, cos, realizzati oltre 120 progetti ecologici (fonte: http://www.iba.nrw.de/main.hhtm). Gli interventi riguardarono la reazione di un sistema di parchi di varie tipologie (culturali, naturali, paesaggistici, ricreativi, ecc...) collegati da una rete ciclopedonale ad altre aree con attrezzature per lo sport e per il tempo libero. Uno di questi parchi, ad esempio, il Duisburg park, sorto su un'aria di due milioni di mq delle ex acciaierie Meiderich della società Thyssen. Il riuso delle acciaierie è stato estremamente originale, con itinerari panoramici che hanno consentito di accedere agli altiforni, alcune pareti degli impianti che sono state trasformate in palestre per free-climbing ed i gasometri che sono diventati piscine per scuole di sub. Il programma previde anche un censimento dei complessi industriali, al fine di inserirli in un piano di restauro di nuove destinazioni d'uso. Abbatterli avrebbe, infatti, comportato un consistente onere finanziario ed una perdita di identità culturale del paesaggio. La loro intrinseca monumentalità è stata, invece, valorizzata da progetti che hanno creato moderne cattedrali post-industriali, adatte ad ospitare eventi artistici e culturali, oltre che nuove attività economiche e produttive. Tra gli interventi ci fu anche il recupero di antichi complessi di abitazioni popolari e la realizzazio- 
ne di nuovi quartieri residenziali. L'innovazione dell'Emscher landscape park è consistita, quindi, non nell'attivazione di politiche economiche ad hoc, ma nella creazione delle condizioni territoriali favorevoli per nuovi investimenti mediante la costruzione di un ambiente capace di competere sui mercati degli investitori con la qualità dello spazio, degli edifici, delle funzioni e dell'offerta culturale, tesa alla valorizzazione degli elementi di pregio che hanno fatto la storia della regione. Nel frattempo, non si può certo dire che l'Emscher park non sia stato un successo con la Ruhr trasformata da caposaldo dell'industria pesante a capitale europea della cultura nel 2010. Oggi la regione presenta, infatti, 3500 monumenti industriali, 200 musei, 120 teatri, 100 centri culturali, 100 sale da concerto e due teatri musicali che ogni anno attirano da tutta la Germania e dal resto dell'Europa milioni di visitatori che vogliono conoscere i progetti IBA e partecipare ai numerosi eventi in programma (fonte: http:// www.ruhr-tourism.de).

Scheda 3 (elaborazione di Placenti F., 2015)

\section{Considerazioni conclusive}

Il valore di un territorio gli viene attribuito non soltanto dalle popolazioni che lo abitano ma, dunque, anche dai turisti che lo frequentano (Martini, 2010). L'esperienza di costruzione di una destinazione turistica deve allora partire proprio dalle esperienze dei turisti ma, anche in un'ottica supply side, senza però tralasciare i più recenti sviluppi del contesto turistico dove, primi fruitori di prodotti e servizi, sono gli abitanti stessi. Il legame tra destinazione ed esperienza del turista si rende quindi essenziale. Ma esiste un concetto standard di esperienza?

L'enciclopedia Treccani definisce l'esperienza come

"la conoscenza diretta, personalmente acquisita con l'osservazione, l'uso o la pratica, di una determinata sfera della realtà”

mentre per Rossi e Goetz (2011, pp. XV-XVI) 
non esiste una definizione di esperienza turistica che possa essere condivisa da tutti, proprio perché l'esperienza è una percezione che si forma nella mente del turista mettendo a confronto le sue aspettative, ovvero l'immagine interiore di ogni viaggio che si forma sulla base di una personale chiave di lettura (valori, motivazioni, propensioni), modificata dall'insieme degli stimoli che esercitano un'influenza su tale immagine e confrontata con quanto realmente vissuto da lui o dalla sua rete relazionale

Nel determinare l'esperienza è quindi necessario il punto di vista del viaggiatore, perché l'aspetto tangibile della stessa scompare nel tempo, mentre rimane indelebile il ricordo dell'evento.

La costruzione dell'offerta turistica per una destinazione non può e non deve più seguire i principi di un vecchio modello fondato sulla standardizzazione dei prodotti. Sia chi progetta (consum-autori) sia chi esperisce (consum-attori) deve seguire logiche volte al potenziamento ed introduzione di servizi innovativi che si basino sempre più sulla ricerca dell'esperienza, della convivialità. Le imprese e le organizzazioni che desiderano intercettare l'interesse e la disponibilità ad acquistare dei consumatori del mondo di oggi devono non più produrre solo beni e/o servizi, ma devono offrire esperienze tali da coinvolgere il viaggiatore a "livello emotivo, fisico, intellettuale o anche spirituale” (Pine e Gilmore, 2000, p. 14). In altre parole

ci si chiede fino a che punto l'approccio degli operatori di una destinazione, nell'allestire una propria offerta, sia capace di stimolare la mente delle persone che visitano quella destinazione attraverso il coinvolgimento di tutti i cinque sensi e non soltanto di quello visivo (Hazel Hahn, 2012, p. 196)

Si rischia però in questo modo che l'espressione culturale di una popolazione, per via di globalizzazione prima e gentrificazione poi, diventi un prodotto omologato con deboli richiami all'identità propria, spesso finendo per rappresentare folkloristicamente solo alcuni aspetti identificati a livello internazionale quali "autentici”, senza che lo siano realmente. Si comprende allora perché sia necessario che nelle agende politiche ai diversi livelli istituzionali siano presenti azioni concrete volte a sviluppare il turismo ed a rafforzare le condizioni territoriali che ne rendono possibili lo sviluppo. Alcune tra queste azioni, volte a scongiurare possibili elementi di crisi quali appena citati, tendono a: 
- Promuovere e sostenere la coesione sociale e territoriale della comunità locali - creazione di capitale sociale territoriale - al fine di incrementare l'attività turistica dei luoghi, rafforzando il valore identitario e costruendo intorno ad esso un processo di convergenza territoriale, tramite pratiche relazionali positive che accrescano i livelli di coesione sociale e di fiducia reciproca (Becattini, 2000);

- Sostenere l'integrazione tra le attività turistiche e il resto dell'economia rurale, creando relazioni sinergiche con altri settori produttivi e con le altre componenti territoriali (Pollice, 2012a);

- Superare il conflitto tra benefici economici e costi ambientali. Doveroso il riferimento al superamento delle soglie di carico del territorio, e sociali, dovuto a forme eccessivamente invasive di turismo che potrebbero innescare processi di deterritorializzazione irreversibili e compromissori dello sviluppo del contesto territoriale;

- Mettere in valore e a sistema, l'insieme di peculiarità paesaggistiche, storico-monumentali ed enogastronomiche che le contraddistinguono.

Queste sono alcune delle tematiche che dovrebbero guidare le scelte volte a promuovere lo sviluppo del turismo, cercando nel contempo di non dimenticare che il turismo rappresenta solo uno degli assi di sviluppo dei territori, posto che il modello di riferimento rimane quello di uno sviluppo integrato incentrato sulla multifunzionalità (Società Geografica Italiana, 2012).

Per concludere, al netto di tutte le considerazioni, una destinazione turistica diventa quindi tale solo nel momento in cui si apre al mercato, ai turisti, ed interagisce con essi al punto che il valore della meta turistica è definita proprio attraverso un processo congiunto di creazione e produzione collaborativa di significati e di risultati. Si prescinde il successo attraverso la condivisione di risorse operand e operant di tutti gli attori coinvolti, valorizzando e tutelando le risorse che più di tutte, in questa nuova prospettiva dell'accoglienza, sono essenziali: le popolazioni.

\section{Referencias}

AHMED, Z.U. “The influence of the components of a state's tourist image on product positioning strategy". Tourism Management, n. 4, v. 12, pp. 331-40, 1991. 
AHUJA, J.; WEBSTER, J. "Perceived disorientation: an examination of a new measure to assess web design effectiveness". In Interacting with computers, v. 14, pp. 15-29, 2001.

AMODIO, T. Turismo e territorio. In BENCARDINO, F. E PREZIOSO, M. (a cura di), Geografia del turismo. Milano, McGraw-Hill, pp. 60-69, 2007.

ATZENI, M. et al. La definizione del concetto di autenticità nelle destinazioni turistiche: un approccio fondato sul laddering, Micro\&Macro Marketing, 2018.

BAGGIO, R. "Collaboration and cooperation in a tourism destination: a network science approach". Curret Issues in Tourism, v. 14, n. 2, pp. 183-89, 2011.

BAGNOLI, L. Manuale di geografia del turismo. Dal Grand Tour ai sistemi turistici. Torino: Utet, 2010.

BALOGLU, S. "McCleary K. W., A model of destination image”. Annals of Tourism Research, v. 26, n. 4, pp. 868-897, 1999.

BARBETTA, G. et al. (a cura di). Distretti culturali: dalla teoria alla pratica. Bologna, Il Mulino, 2013.

BARCA, F. et al. "The case of regional development interventation: place-based versus place-neutral approaches". In: Journal of Regional Science, v. 52 , n. 1, pp. 134-52, 2012.

BARILE, S. e SAVIANO, M. L. "A new perspective of system complexity in service science". Impresa Ambiente Management, v. 4, n. 3, pp. 375-414, 2010.

BAUMAN, Z. Dentro la globalizzazione, Le conseguenze sulle persone. Laterza, Roma-Bari, 1999a.

BECATTINI, G. "Lo sviluppo locale nel mercato globale. Riflessioni controcorrente". In: La Questione Agraria, vol. 1, pp. 13-27, 2000.

BELL, D. e JAYNE, M. (a cura di). City of wuarters: Urban villages in the contemporary city. Ashgate, Aldershot, 2004.

BELLETTI, G. "Ruralità e turismo”. Agriregionieuropa, anno 6, 20, 2010. 
BERMAN, M. The politics of authenticity. London: Allen, 1970.

BIGNÈ, et al. "Tourism image, evaluation variables and after purchase behaviour: inter-relationship”, Tourism Management, v. 22, n. 6, pp. 607-16, 2001.

BRUNER, E. M. Culture on Tour: ethnographies of travel. University of Chicago Press, Chicago-London, 2004.

BUTLER, R. W. "The concept of a tourist area cycle of evolution: implications for management of resources". In: Canadian Geographer, v. 24, n. 1, pp. 5-12, 1980.

CANELA, G. e FIGINI, P. Economia dei sistemi turistici. Milano, McGraw-Hill, 2005.

CARRUBBO, L. La co-creazione di valore nelle destinazioni turistiche. Roma, Rirea, 2013.

CARRUS, P. P. e MELIS, G. "L'innovazione delle scelte strategiche in condizioni di crescente complessità. Risorse, processi e competenze per la creazione di valore nel nuovo scenario". Franco Angeli, v. 122, 2006.

CELLINI, R. "Il turismo nei programmi di crescita e sviluppo: analisi teoriche ed esperienze”. In LA ROSA, R. (a cura di). Lo sviluppo del turismo in Sicilia. Milano: Franco Angeli, 2004.

CENSIS. $5^{\circ}$ rapporto Censis/Ucsi sulla comunicazione in Italia, disponibile su www. censis.it, 2005.

CIRELLI, C. (a cura di). Gli spazi del commercio nei processi di trasformazione urbana. Bologna, Pàtron, 2007.

CIRELLI, C. et al, E. Percorsi creativi di turismo urbano. I luoghi dell'entertainment nella città del tempo libero. Bologna, Pàtron, 2013.

CIRELLI, C. e NICOSIA, E. Il centro storico, centro commerciale naturale. In: SCANU, G., Paesaggi, ambiente, culture, economie. La Sardegna nel mondo mediterraneo. Bologna, Pàtron, pp. 201-18, 2013.

CUSIMANO, G. (a cura di). Il turismo nelle/delle destinazioni. Bologna, Pàtron Editore, 2015. 
DALL'ARA, G. “I Sistemi turistici locali: una opportunità di sviluppo". In: DALL'ARA, G. e MORANDI, F. (a cura di). I sistemi turistici locali. Normativa, progetti e opportunità. Matelica, Halley Editore, pp. 83-112, 2006.

DALLI, D. "Produzione, distribuzione e appropriazione del valore di mercato: il ruolo del consumatore". International Marketing Trends Conference, 21-23 gennaio. Venezia, 2010.

DE VELLIS, R. F. “Scale development: theory and applications”. Sage pubblications, Fourth Edition, vol. 26, 2016.

DEL BALDO, M. "Contratto di rete e turismo: la collaborazione aziendale come driver della competitività del settore e del territorio. Un'analisi delle prime esperienze”. In: Rivista di Scienze del turismo, v. 2, pp. 71-116, 2014.

DELL'AGNESE, E. Media e geopolítica. In: LIZZA, G. (a cura di). Geogpolitica delle prossime sfide. Torino, Utet, pp. 243-273, 2011.

DELLA CORTE, V. e SCIARELLI, S. La gestione dei sistemi locali di offerta turística. Padova, Cedam, 2000.

DI FELICE, G. Da "luoghi letterari a luoghi turistici nella mediazione cinematografica. Due casi internazionali a confronto". In CIRELLI, C. et al (a cura di). Percorsi creativi di turismo urbano - Creative paths of urban tourism. I luoghi dell'entertainment nella città del tempo libero. Bologna, Pàtron, pp. 337-52, 2013.

EJARQUE, J. e LE DMO, E. “Gli enti del turismo italiani sono destinati a sparire?” Fourtourism blog http://www.fourtourismblog.it/le-dmo-gli-enti-del-turismo-italiani-destinati-sarire, 2016.

ENIT. Programma esecutivo annuale, 2005, www.enit.it .

EVANS, C. "Creative spaces, tourism and the city". In RICHARDS, G. e WILSON, J. Creativity, tourism and development. Routledge, Londra-New York, pp. 57-72, 2007.

FABRIS, G. Il nuovo consumatore: verso il postmoderno. Milano, Franco Angeli, 2003.

FRIAS, J.D. M. et al. "The effect of value-creation on consumer-based destination brand equity". Journal of Travel Research, v. 56, n. 8, pp. 1011-31, 2017. 
GARTNER, W. C. "Image formation process".Journal of Travel and Tourism Marketing, v. 2, n. 3, pp. 191-215, 1993.

GETZ, D. Tourism planning and research. Tradition, models and futures. In: HALL, M. C. e PAGE, S. J. (eds. 1999). The geography of tourism and recreation. Enironment, place and space. New York, Routledge, 1987.

GIANNONE, M. La componente territoriale nei sistemi turistici locali. In: DALL'ARA, G. e MORANDI, F. (a cura di). I sistemi turistici locali. Normativa, progetti e opportunità. Matelica, Halley Editore, pp. 71-81, 2006.

GOLINELLI, C. M. Il territorio sistema vitale: verso un modello di analisi. Torino, Giappichelli, 2002.

et al. Governo e marketing del territorio. Sinergie, Rapporti di ricerca, v. 2, n. 23, pp. 17-34, 2006.

. L'approccio sistemico al governo dell'impresa: Valorizzazione delle capacità, rapporti intersistemici e rischio nell'azione di governo. Padova: Cedam, 2000.

GRAZIANO, T. Dai migranti ai turisti. Gentrification, luoghi del consumo e modelli di fruizione nelle città globali. Roma: Aracne, 2013.

GUATRI, L. La teoria di creazione del valore. Una via europea. Milano: Egea, 1991.

HALL, C. M. Tourism planning. Policies, processes and relationship. Pearson: Harlow, 2000.

HAZEL, H. H. The Flâneur, the Tourist, The Global Flâneur, and magazine reading as Flâneur. Dix Neuf, v. 16, n. 2, pp. 193-210, 2012.

ID. Dal marketing territoriale alla gestione competitiva del territorio. Napoli, Edizioni Scientifiche Italiane, 2000.

ID. La società dell'incertezza. Bologna, Il Mulino, 1999b.

. Leconomia delle esperienze. Milano, Etas, 1999. 
. Piccole destinazione e marketing. Officina Turistica, 2 maggio, http://www. officinaturistica.com/2016/05/piccole-destinazioni-marketing/, 2016.

. "Sistemi territoriali e apprendimento localizzato". In BIGGIERO, L. e SAMMARRA, A. (a cura di). Apprendimento, identità e marketing del territorio. Roma: Carocci, pp. 36-56, 2002.

KOLTER, P. Marketing management. Milano: McGraw-Hill, 2004.

LEIPER, N. “Tourist attraction system”. In Annals of tourism research, v. 17, n. 3, pp. 367-84, 1990.

LIN, N. Social Capital: a theory of social structure and action. Cambridge, Cambridge University Press, 2001.

LUSCH, R. F. e VARGO, S. L. The service-dominant logic of marketing: Dialog, debate, and directions. Routledge, Londra, 2014.

LUSH, R. F. e VARGO, S. L. Service-dominant logica s a foundation for a general theory. The service-dominant logicof marketing: Dialog, debate, and directions, 406, 2006.

MARTINI, U. Destinazione turistica e territorio. In: FRANCH, M. Marketing delle destinazioni turistiche: metodi, approcci estrumenti. Milano: McGraw-Hill, 2010.

MATURANA, H. Autocoscienza e realtà. Milano: Rafaello Cortina Editore, 1993.

MCCOOL, S. F. e LIME, D. W. “Tourism Carryn Capacity. Tempting Gantasy or Useful Really?”. In: Journal of Sustainable Tourism, v. 9, n.5, pp. 372-88, 2000.

MOSCARDO, G. "Shopping as a destination attraction: An empirital examination of the role of shoppung in tourists destination choice and experience". In: Journal of vacation Marketing, v. 10, n. 4, pp. 294-307, 2004.

NAPOLITANO, M. R. Comportamento e soddisfazione del turista. I risultati di una ricerca in Campania. Milano: Franco Angeli, 2010.

NAPOLITANO, M. R. e DE NISCO, A. Marketing turistico e destination branding. Lezioni da alcune esperienze internazionali. In: BENCARDINO, F. e PREZIOSO, M. (a cura di). Geografia del turismo. Milano: McGraw-Hill, pp. 253-84, 2007. 
PECHLANER, H. e WEIERMAIR, K. Destination management. Fondamenti di marketing gestione delle destinazioni turistiche. Milano, Touring Club Italiano, 2000.

PEZZANO, A. "DMO fai la cosa giusta?", Officina Turistica, 20 ottobre, http://www. officinaturistica.com/2015/10/dmo-fai-la-cosa-giusta/, 2015.

PIKE, S. e RYAN, C. "Destination positioning analysis through a comparison of cognitive, affective, and conative perceptions”. In: Journal of Travel Research, v. 44, pp. 333-42, 2004.

PIKKEMAAT, B. “The measurement of destination image: the case of Austria”. In: The Poznan University of Economics Review, v. 41, n. 1, pp. 87-102, 2004.

PINE, B. J. e GILMORE, J. H. Oltre il servizio. L'economia delle esperienze. Milano, Etas, (ed. or. 1999), 2000.

PLACENTI, F. "Distretti culturali e turismo. Il caso dei distretti culturali siciliani tra aspettative disattese ed opportunità mancate”. In CUSIMANO, G. (a cura di). Il turismo nelle/delle destinazioni. Bologna, Pàtron Editore, 2015.

POLLICE, F. "I sistemi turistici agro culturali. Configurazioni territoriali ed orientamenti competitivi”. In: Annali del Turismo, Geoprogress Edizioni, v. 1, Novara, 2012a.

RANJAN, K. R. e READ, S. "Value co-creation: concept and measurement". Journal of the Academy of Marketing Science, v. 44, n. 3, pp. 290-315, 2016.

ROCCA, G. Dal prototurismo al turismo globale. Momenti, percorsi di ricerca, casi di studio. Torino, Giappichelli, 2013.

ROSSI, A. e GOETZ, M. Creare offerte turistiche vincenti con tourist experience design. Milano, Hoepli, 2011.

ROSSI, C. Marketing collaborativo e online value co-creation. Limpresa e la sfida del consumatore produttivo, ranco. Milano, Angeli, 2014.

RULLANI, E. La fabbrica dell'immateriale. Roma: Carocci, 2004. 
SABEL, C. F. "Regionalismo sperimentale e i dilemmi della politica economica regionale in Europa”. In PERULLI, P. (a cura di). Neoregionalismo. L'economia-arcipelago. Torino: Bollati Boringhieri, pp. 70-110, 1998.

SOCIETÀ GEOGRAFICA ITALIANA. Rapporto annuale 2012. I nuovi spazi dell'agricoltura italiana. Roma, SGI, 2012.

SONMEZ, S. e SIRAKAYA, E. "A distorted destination image? The case of Turkey". Journal of Travel Research, v. 41, n. 2, pp. 185-96, 2002.

TAGHIZADEH, S. K. et al. "Scale development and validation for DART model of value co-creation process on innovation strategy". Journal of Business\& Industrial Marketing, v. 31, n. 1, pp. 24-35, 2016.

TAMMA, M. Destination Management: gestire prodotti e sistemi locali di offerta. In: FRANCH, M. (a cura di).Destination Management. Governare il turismo fra locale e globale. Torino, Giappichelli, 2002.

THOMAS, R. e WOOD, E. “Innovation in tourism: re-conceptualising and measuring the absorptive capacity of the hotel sector". Tourism Management, v. 45, pp. 39-48, 2014 .

VALDANI, E. e ANCARANI, F. Strategie di marketing del territorio. Milano: Egea, 2000.

VARGO, S. L. e LUSH, R. F. “Evolving to a new dominant logic for marketing”. In: Journal of marketing, v. 68, n. 1, pp. 1-17, 2004.

VICARI, S. “Conoscenza e impresa”. Sinergie, n. 76, pp. 44-66, 2008. 